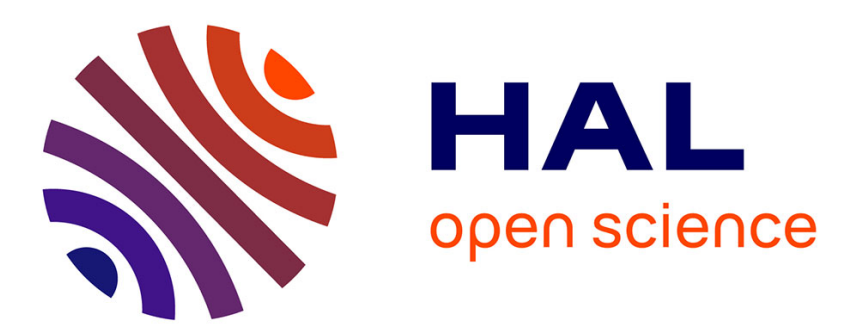

\title{
Fault-tolerant final approach navigation for a fixed-wing UAV by using long-range stereo camera system
}

Yoko Watanabe, Augustin Manecy, Alexandre Amiez, Shin Aoki, Sho Nagai

\section{To cite this version:}

Yoko Watanabe, Augustin Manecy, Alexandre Amiez, Shin Aoki, Sho Nagai. Fault-tolerant final approach navigation for a fixed-wing UAV by using long-range stereo camera system. ICUAS 2020: International conference on Unmanned Aircraft Systems, Sep 2020, Athènes, Greece. hal-03211158

\section{HAL Id: hal-03211158 \\ https://hal.science/hal-03211158}

Submitted on 28 Apr 2021

HAL is a multi-disciplinary open access archive for the deposit and dissemination of scientific research documents, whether they are published or not. The documents may come from teaching and research institutions in France or abroad, or from public or private research centers.
L'archive ouverte pluridisciplinaire HAL, est destinée au dépôt et à la diffusion de documents scientifiques de niveau recherche, publiés ou non, émanant des établissements d'enseignement et de recherche français ou étrangers, des laboratoires publics ou privés. 


\title{
Fault-tolerant final approach navigation for a fixed-wing UAV by using long-range stereo camera system
}

\author{
Yoko Watanabe ${ }^{1}$, Augustin Manecy ${ }^{1}$, Alexandre Amiez ${ }^{1}$, Shin Aoki ${ }^{2}$ and Sho Nagai ${ }^{2}$
}

\begin{abstract}
This paper presents an onboard vision/GNSSintegrated fault tolerant navigation system for aircraft final approach. The world-first long-range stereo camera system which captures an object and measures its distance up to 1 $\mathrm{km}$ has been developed, and implemented for runway feature detection. The navigation filter is designed based on timedelayed Error State Kalman filter for tightly coupling GNSS pseudo-range measurements with the stereo camera outputs. Then it is augmented with an integrity monitoring function for fault detection and protection level calculation. The whole navigation system has been evaluated on real sensor data sets, acquired on the ONERA experimental UAV, with GNSS fault emulation. The results show an improvement both in fault detectability and in navigation performance thanks to adding the stereo camera to the conventional GNSS navigation setup.
\end{abstract}

\section{INTRODUCTION}

Increasing an autonomy level of the aircraft flight control system will alleviate the pilots' task and stress in handling critical situations, and hence is an important key to enhance civil aviation safety. In order to contribute towards it, a Europe-Japan collaborative research project called VISION (Validation of Integrated Safety-enhanced Intelligent flight cONtrol) has been carried out in 2016-2019[1]. This project aimed at developing and flight-evaluating advanced aircraft Guidance, Navigation and Control (GNC) solutions that can automatically detect and overcome some critical flight situations. As a part of the VISION project, ONERA -The French Aerospace Laboratory- and a Japanese well-known photonics company RICOH Co., Ltd. have jointly developed a fault-tolerant navigation system for aircraft final approach by using a long-range stereo camera, and realized it onboard a fixed-wing UAV platform called K50-Advanced (Fig.1) manufactured within the project.

Using onboard vision sensors in aircraft final approach and landing navigation is a very natural idea, and have been addressed in [2] and [3] for civil aviation application. [3] evaluated the image feature-based glide path estimation algorithm in a high fidelity aircraft simulator. There are also many work in vision-based autonomous UAV landing, and some actually flight tested the navigation and guidance approaches in a closed-loop manner[4]. These "vision-only" approaches use onboard vision as an alternative, but not additional, navigation sensor to the classical ones such as GNSS (Global Navigation Satellite Systems). Unlike those,

\footnotetext{
${ }^{1}$ Research engineers at Department of Information Processing and Systems, ONERA - The French Aerospace Laboratory, 31055 Toulouse, France. ffirst name\}.\{last name\}eonera.fr

${ }^{2}$ Research engineers at Applied Optics System Research Center / R\&D Division, RICOH Company Ltd., 243-0460 Ebina, Japan.

ffirst name $\}$. last name\}@jp.ricoh.com
}

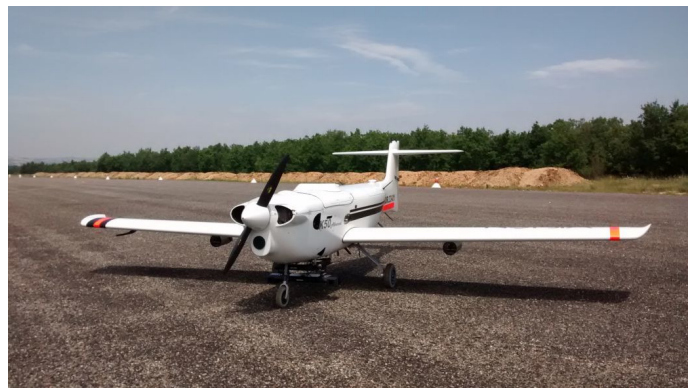

Fig. 1. K50-Advanced UAV platform (fuselage length $=3 \mathrm{~m}$, wingspan $=$ $4 \mathrm{~m}$, maximum take-off weight $=60 \mathrm{~kg}$, useful payload $=20 \mathrm{~kg}$, loiter speed $=72 \mathrm{~km} / \mathrm{h}$, stall speed $=65 \mathrm{~km} / \mathrm{h}$ ) with the RICOH long-range stereo camera under its fuselage.

[5] has proposed a vision-augmented automatic landing system and flight-demonstrated it on a real aircraft. It uses vision information as a dissimilar reference in integrity monitoring, but not directly in the navigation filter, only for detecting GNSS failures.

Combining the both ideas, this paper proposes a tightlycoupled vision/GNSS navigation system for aircraft final approach, augmented with an integrity monitoring function for sensor fault detection and protection level calculation. For this purpose, RICOH has developed the world-first longrange stereo camera system (Fig.2) which is able to detect runway marker positions and measures their distances from up to $1 \mathrm{~km}$ away on a final approach trajectory. This stereo camera system has been implemented onboard the K50 platform, and its real-time performance was evaluated through flight experiments. The navigation filter to tightly fuse the stereo camera outputs with other sensor measurements is designed based on time-delayed Kalman filter (KF)[6] in order to handle a non-negligible delay of the vision measurements due to image processing time. It benefits from image trigger signals of the camera device, and performs the delayed KF process in an efficient manner[7]. Then an integrity monitoring (IM) function is founded by applying a classical Least Square(LS)-RAIM (Receiver Autonomous Integrity Monitoring) algorithm, widely applied in GNSS receivers, on a batch-realization of this filter design[8]. An IM reset mechanism is also introduced for re-initializing a fault detector regularly.

The proposed fault-tolerant navigation system has been tested on real sensor data sets (including GNSS pseudorange measurements and the RICOH stereo camera outputs calculated in real time), acquired onboard the K50 UAV on final approach trajectory. GNSS fault is emulated on one 
selected satellite measurement to evaluate the IM capability. The test results confirm that adding the stereo camera information improves both the fault detectability and the navigation performance.

\section{LONG-RANGE STEREO CAMERA SYSTEM}

\section{A. Stereo Camera System}

The long-range stereo camera system (Fig.2) which enables real-time distance measurement to capture objects up to 1,000 meters away was developed by RICOH Co., Ltd. The specification of this stereo camera system is shown in Table I. Two identical monochrome image sensors of $4096 \times 3000$ pixel resolution were positioned parallel to each other to obtain right and left images. The camera system issues synchronized triggers to the two image sensors and recorded images from each. The developed stereo camera system computes a depth image using an FPGA logic component to compare the right and left images. Then, it estimates the camera pose (position and orientation), w.r.t. the runway, from the stereo camera monochrome images and the depth image in real time. The camera component which connects between the right and left camera was made of CFRP (Carbon Fiber Reinforced Plastics) to mitigate vibration effect during taxiing, landing and flight. Fig.3 shows an example of the right camera image and the depth image captured onboard the K50 UAV during its final approach at ONERA Septfonds flight test field using this stereo camera system.

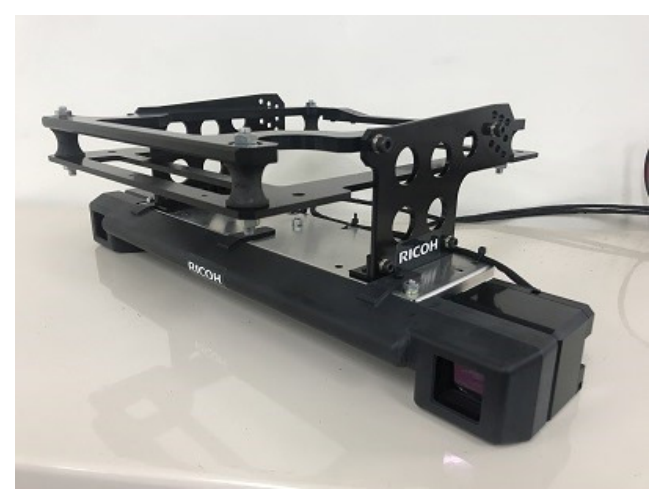

Fig. 2. Long-range stereo camera system

TABLE I

SPECIFICATION OF LONG-RANGE STEREO CAMERA SYSTEM

\begin{tabular}{ll}
\hline Optics & RICOH's original design \\
Resolution(pixel) & $4086 \times 3000$ \\
Chroma & Monochrome \\
Shutter & Global shutter \\
Baseline & $400 \mathrm{~mm}$ \\
FOV & $22.8^{\circ} \times 16.8^{\circ}$ \\
Frame Rate & $15 \mathrm{FPS}$ \\
\hline
\end{tabular}

\section{B. Runway Feature Detection}

Estimation of the aircraft pose with respect to the runway by the stereo camera system uses image position and disparity information of the runway threshold line and centerline,

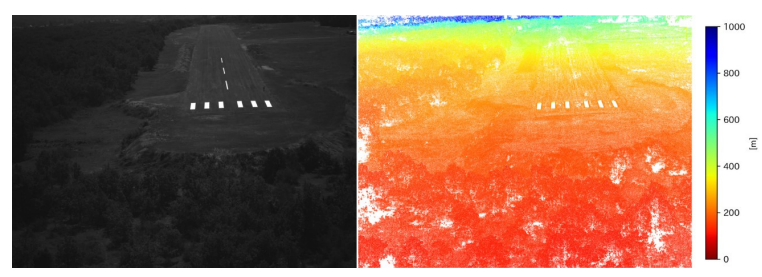

Fig. 3. Example of the right camera image and the depth image

as well as a vanishing point calculated from sidelines. The goal of preliminary image processing is to extract the above image features. The algorithm assumes to use a dimension of runway marking which is shown in Fig.4.

\section{1) Runway detection:}

a) Cascade classifier: An apparent shape of the runway marking on the stereo camera images varies depending on changes in the aircraft pose. Especially its size and orientation on images are quite different under changes in the aircraft approach distance and roll angle. In case of using template matching for runway marking detection, a lot of template images are mandatory to support the above changes of the runway shape on image. Therefore, our algorithm uses a cascade classifier[10] not to have a lot of template images and correspond to above-mentioned changes of shape on image. Cascade classifier learns from a lot of simulated images of the runway marking shown in Fig.4 under different aircraft poses through assumed final approach flight path.

b) Restriction of search area: As the aircraft maneuverability (velocity and angular rate) is limited, it is reasonable to think that fluctuation of the runway marking position and size on a current image from those detected on a previous image has a restriction. In case runway features are captured on an image for the first time, the algorithm searches them on a whole area of image. Otherwise, it searches them only on an image area near their positions detected on the previous image in order to reduce the processing time.

\section{2) Threshold line detection:}

a) Edge filter: In our runway detection process, a rectangle region neighboring the runway threshold line is extracted. It can be assumed that a main object in this rectangle region is the threshold bars. Therefore, a simple horizontal edge detection filter (vertically aligned 3 pixels

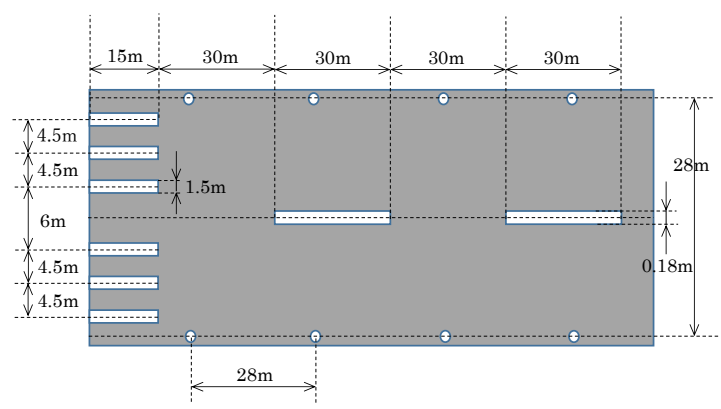

Fig. 4. Dimension of runway marking 


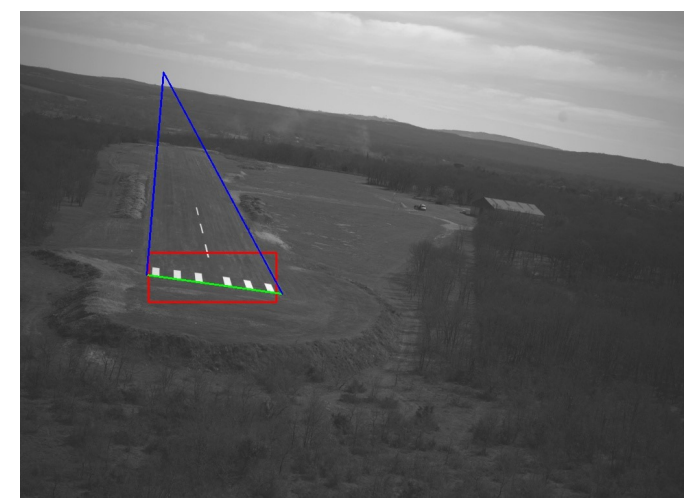

Fig. 5. Example of runway detection results: (Red) Region of runway end, (Green) Threshold line, (Blue) Sidelines and vanishing point.

with weights $1,0,-1$ ) is applied to extract bottom edge pixels of the threshold bars from this region.

b) Line fitting: Line fitting is processed by using a model fitting method RANSAC (RANdom SAmple Consensus)[11] which is robust for noisy outliers to edge candidate pixels which are extracted from image by the above-mentioned edge filter.

3) Vanishing point detection: Vanishing point, where image projection of lines parallel to the runway axis appears to converge, can be detected by the following two methods.

a) Method based on centerline: The runway centerline can be detected by the same line fitting method used for the threshold line detection. It uses a vertical edge detection filter around a midpoint of the threshold line on image. Then, it collects disparity information of the centerline pixels and estimates a vanishing point on an extended centerline by disparity zero which corresponds to a point at infinity.

b) Method based on sidelines: As shown in Fig.4, white cones lined on both right and left sides of the runway are used as sideline marking in this algorithm. It extracts cone candidate pixels for obtaining a bottom center position of each white cone which touches the runway surface based on a fact that white cones are relatively brighter than the runway surface. Fig. 6 shows an extraction example of the bottom center of a white cone. The pixel marked as "C" is a pixel-of-interest with its intensity $I_{C}$. Each of 3 pixels marked as "L", "R" and "B" shows a compared region, of which average intensity is $\overline{I_{L}}, \overline{I_{R}}$ and $\overline{I_{B}}$. A white cone candidate pixel is extracted in case the intensity of the pixelof-interest inflated with a constant factor $\alpha$ is higher than the average intensity of the compared regions as follows.

$$
\left(\alpha I_{C}>\overline{I_{L}}\right) \text { and }\left(\alpha I_{C}>\overline{I_{R}}\right) \text { and }\left(\alpha I_{C}>\overline{I_{B}}\right)
$$

The extracted white cone pixels are processed by RANSAC to extract the sidelines.

The centerline-based method performs faster than the sideline-based one since line fitting is processed only once. However, the estimation result of disparity zero is not so accurate. For optimization of real time image processing, vanishing point detection by sidelines with good accuracy

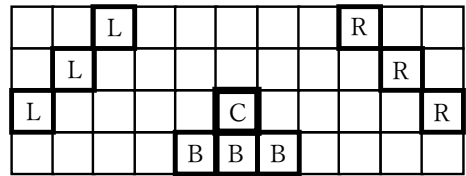

Fig. 6. Extraction example of bottom center of white cone: (C) Pixel-ofinterest, (L,R and B) Compared regions.

is used in case the runway is successfully detected on the previous image because there is a margin of process time thanks to the search area restriction. Otherwise, the centerline-based method with short process time is used since the runway detection process needs to consume time.

4) Validation: The image position and disparity of the extracted runway features are validated if they lie within their assumed range calculated from the results on the previous image. In addition, they are also validated in a mid-process of the runway detection by using parameters like the number of valid disparity pixels in order to remove detection error. This validation process avoids that the misrecognition continually occur on subsequent frames once it occurs on one frame due to the wrong search range selection.

\section{NAVIGATION SYSTEM DESIGN}

\section{A. Estimation Filters}

The attitude and position estimation filters are designed independently, both based on the time-delayed Error State Kalman Filter (ESKF)[7].

1) Reference frames: Different reference frames are defined and used in the filter design. Besides the conventionally used ones (WGS84, ECEF, NED, aircraft body), the following frames are defined.

- Runway frame: a Cartesian coordinate system fixed on Earth at a runway threshold center point, with its $X$ axis aligned with the runway axis, and $Z$-axis normal downward to the runway plane. We denote its origin and orientation (in quaternion) w.r.t. the NED frame by $\boldsymbol{X}_{T H D}$ and $\boldsymbol{q}_{R / I}$

- Camera frame: a Cartesian coordinate system fixed on a camera, with its origin at a camera center, its $X$ axis aligned with the optical axis and $Y$-axis with the image horizontal to the right. We denote its origin and orientation (in quaternion) w.r.t. the aircraft body frame by $\Delta \boldsymbol{X}_{C A M}$ and $\boldsymbol{q}_{C / B}$, which are derived from camera extrinsic parameter calibration.

- Image frame: a 2D pixel coordinate system fixed on a camera's image plane with its origin at a left-top corner of the image. A pin-hole camera model transforms a position vector $\boldsymbol{X}_{t}^{C}=\left[\begin{array}{lll}X_{t}^{C} & Y_{t}^{C} & Z_{t}^{C}\end{array}\right]^{T}$ of a target in the camera frame into a pixel coordinates:

$$
\boldsymbol{p}_{t}=\boldsymbol{p}\left(\boldsymbol{X}_{t}^{C}\right)=\left[\begin{array}{ccc}
c_{x} & f_{x} & 0 \\
c_{y} & 0 & f_{y}
\end{array}\right] \frac{\boldsymbol{X}_{t}^{C}}{X_{t}^{C}}=\mathcal{C} \frac{\boldsymbol{X}_{t}^{C}}{X_{t}^{C}}
$$

where a camera matrix $\mathcal{C}$ can be derived from camera intrinsic parameter calibration. 
2) Attitude filter state and measurements: The attitude true state $\boldsymbol{x}_{a_{t}}$ is defined by a quaternion $\boldsymbol{q}_{t}$ representing the UAV's orientation in the NED frame and a gyro bias $\boldsymbol{b}_{\omega_{t}}$ in the aircraft body frame. This true state is divided into the nominal attitude state $\boldsymbol{x}_{a}$ and the error state $\boldsymbol{\delta} \boldsymbol{x}_{a}$. The nominal state evolves from time $t_{k}$ to $t_{k+1}$ with the following deterministic kinematics:

$$
\boldsymbol{x}_{a_{k+1}}=\left[\begin{array}{c}
\boldsymbol{q}_{k+1} \\
\boldsymbol{b}_{\omega_{k+1}}
\end{array}\right]=\left[\begin{array}{c}
\boldsymbol{q}_{k} \otimes \boldsymbol{q}\left(\left(\boldsymbol{\omega}_{I M U_{k}}-\boldsymbol{b}_{\omega_{k}}\right) \Delta t_{k+1}\right) \\
\boldsymbol{b}_{\omega_{k}}
\end{array}\right]
$$

where $\boldsymbol{\omega}_{I M U_{k}}$ is an angular rate measurement from IMU and $\Delta t_{k+1}=t_{k+1}-t_{k}$. The quaternion operation functions (such as $\otimes, \boldsymbol{q}(\cdot)$ ) used in this paper are from [9]. The error state is defined by $\boldsymbol{\delta} \boldsymbol{x}_{a}^{T}=\left[\begin{array}{ll}\boldsymbol{\delta} \boldsymbol{\theta}^{T} & \boldsymbol{\delta} \boldsymbol{b}_{\omega}^{T}\end{array}\right]$ such that

$$
\boldsymbol{x}_{a_{t}}=\boldsymbol{x}_{a} \oplus \boldsymbol{\delta} \boldsymbol{x}_{a}=\left[\begin{array}{l}
\boldsymbol{q} \otimes \boldsymbol{q}(\boldsymbol{\delta} \boldsymbol{\theta}) \\
\boldsymbol{b}_{\omega}+\boldsymbol{\delta} \boldsymbol{b}_{\omega}
\end{array}\right]
$$

It follows the below linear kinematics model.

$$
\boldsymbol{\delta} \boldsymbol{x}_{a_{k+1}}=\left(I+\left[\begin{array}{cc}
-\Omega_{k} & -I \\
O & O
\end{array}\right] \Delta t_{k+1}\right) \boldsymbol{\delta} \boldsymbol{x}_{a_{k}}+\left[\begin{array}{c}
\boldsymbol{\nu}_{\omega_{k}} \\
\boldsymbol{\nu}_{b_{\omega k}}
\end{array}\right]
$$

where $\Omega_{k}=\left[\left(\boldsymbol{\omega}_{I M U_{k}}-\boldsymbol{b}_{\omega_{k}}\right) \times\right]$ is a skew-symmetric crossproduct matrix. $\nu$ 's are the zero-mean Gaussian noise.

Measurements used to correct the attitude estimation during the final approach phase are the vanishing point $\boldsymbol{p}_{v}$ and the runway threshold line inclination angle $\theta_{T H D}$ (Fig. 7) calculated by the stereo camera system. By using (1) and a function $\theta(\boldsymbol{x})=\arctan (y / x)$, these measurements are modeled as follows.

$\boldsymbol{z}_{a}=\left[\begin{array}{c}\boldsymbol{p}_{v} \\ \theta_{T H D}\end{array}\right]+\left[\begin{array}{l}\boldsymbol{\xi}_{v} \\ \xi_{\theta}\end{array}\right]=\left[\begin{array}{c}\boldsymbol{p}\left(\boldsymbol{e}_{R_{X t}}^{C}\right) \\ \theta\left(\left(\mathcal{C}-\boldsymbol{p}_{T H D} \boldsymbol{e}_{1}^{T}\right) \boldsymbol{e}_{R_{Y t}}^{C}\right)\end{array}\right]+\boldsymbol{\xi}_{a}$ where $\boldsymbol{e}_{R_{(X, Y)_{t}}^{C}}^{C}=R\left(\boldsymbol{q}_{C / B}^{*} \otimes \boldsymbol{q}_{t}^{*} \otimes \boldsymbol{q}_{R / I}\right) \boldsymbol{e}_{(1,2)}$ is the runway $X$ - and $Y$-axis directions in the camera frame. $\xi$ 's are zeromean Gaussian measurement errors.

3) Position filter state and measurements: Let $\boldsymbol{x}_{p_{t}}$ be a true position state vector defined by

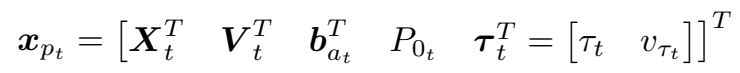

where $\boldsymbol{X}$ and $\boldsymbol{V}$ are the UAV position and velocity in the NED frame, $\boldsymbol{b}_{a}$ is an accelerometer bias in the aircraft body frame, $P_{0}$ is a pressure adjusted to a standard atmosphere at sea level and $\tau$ is a clock offset of a GNSS receiver and

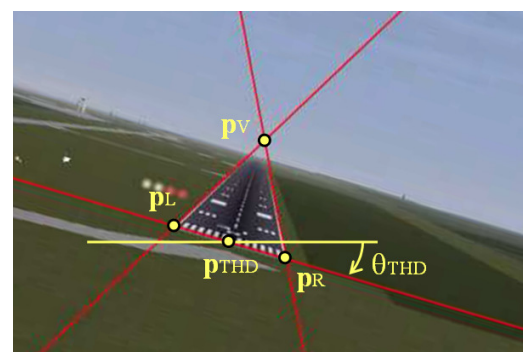

Fig. 7. Runway image feature measurements used in the navigation filter: $\boldsymbol{p}_{v}=$ Vanishing point pixel coordinates, $\theta_{T H D}=$ Threshold line inclination, $\boldsymbol{p}_{T H D}=$ Threshold center pixel coordinates its changing rate. Similarly as done to the attitude state, the nominal position state is defined so that it follows the following deterministic kinematics.

$$
\boldsymbol{x}_{p_{k+1}}=\boldsymbol{x}_{p_{k}}+\left[\begin{array}{c}
\boldsymbol{V}_{k} \Delta t_{k+1}+\frac{1}{2} \boldsymbol{a}_{k} \Delta t_{k+1}^{2} \\
\boldsymbol{a}_{k} \Delta t_{k+1} \\
\mathbf{0} \\
0 \\
{\left[\begin{array}{c}
v_{\tau k} \Delta t_{k+1} \\
0
\end{array}\right]}
\end{array}\right]
$$

where $\boldsymbol{a}_{k}=R\left(\boldsymbol{q}_{k}\right)\left(\boldsymbol{a}_{I M U_{k}}-\boldsymbol{b}_{a_{k}}\right)+\boldsymbol{g}$ is the acceleration estimate with the IMU accelerometer measurement $\boldsymbol{a}_{I M U}$ and a gravity vector $\boldsymbol{g}$. The error state $\boldsymbol{\delta} \boldsymbol{x}_{p}$ is defined s.t.

$$
\boldsymbol{x}_{p_{t}}=\boldsymbol{x}_{p} \oplus \boldsymbol{\delta} \boldsymbol{x}_{p}=\left[\begin{array}{c}
\boldsymbol{X}+R(\boldsymbol{q}) \boldsymbol{\delta} \boldsymbol{X} \\
\boldsymbol{V}+R(\boldsymbol{q}) \boldsymbol{\delta} \boldsymbol{V} \\
\boldsymbol{b}_{a}+\boldsymbol{\delta} \boldsymbol{b}_{a} \\
P_{0}+\delta P_{0} \\
\boldsymbol{\tau}+\boldsymbol{\delta} \boldsymbol{\tau}
\end{array}\right]
$$

Then it follows a linear kinematics:

$$
\boldsymbol{\delta} \boldsymbol{x}_{p_{k+1}}=\left(I+F_{k} \Delta t_{k+1}\right) \boldsymbol{\delta} \boldsymbol{x}_{p_{k}}+G_{k} \Delta t_{k+1} \boldsymbol{\delta} \boldsymbol{\theta}_{k}+\boldsymbol{\nu}_{p_{k}}
$$

where, with $\mathcal{A}_{k}=\left[\left(\boldsymbol{a}_{I M U_{k}}-\boldsymbol{b}_{a_{k}}\right) \times\right]$ being a skewsymmetric cross-product matrix,

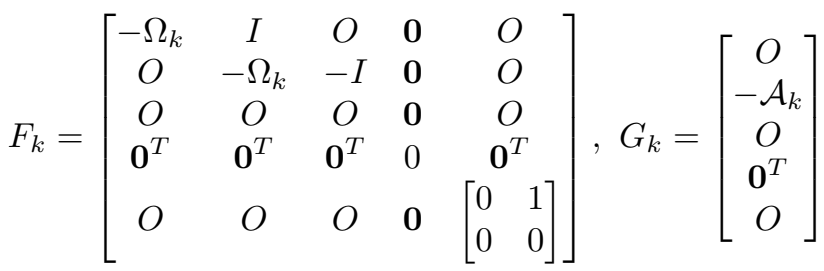

Three sensors are used to correct the position estimate: GNSS, barometer and the stereo camera. The proposed navigation filter tightly integrates GNSS with the stereo camera by taking pseudo-range measurements of each visible satellite. The GNSS pseudo-range measurements are corrected with ionospheric/tropospheric delays and satellite clock offset provided by SBAS (Satellite-Based Augmentation System) signals. After these corrections, the $i$-th satellite pseudo-range measurement is modeled by

$$
\begin{aligned}
z_{\rho_{i}} & =\rho_{i}+\xi_{\rho_{i}}=\left\|\boldsymbol{X}_{G N S S_{t}}^{E C E F}-\boldsymbol{X}_{S A T_{i}}^{E C E F}\right\|+c \tau_{t}+\xi_{\rho_{i}} \\
& =\left\|\boldsymbol{X}_{t}+R\left(\boldsymbol{q}_{t}\right) \Delta \boldsymbol{X}_{G N S S}-\boldsymbol{X}_{S A T_{i}}^{N E D}\right\|+c \tau_{t}+\xi_{\rho_{i}}
\end{aligned}
$$

where $\boldsymbol{X}_{S A T_{i}}^{E C E F}$ is a position of the $i$-th satellite in the ECEF frame, provided by the receiver, $\Delta \boldsymbol{X}_{G N S S}$ is a GNSS antenna position in the UAV body frame and $c$ is a light speed. The GNSS velocity measurement is directly given in the NED frame:

$$
\boldsymbol{z}_{V}=\boldsymbol{V}_{G N S S_{t}}+\boldsymbol{\xi}_{V}=\boldsymbol{V}_{t}+R\left(\boldsymbol{q}_{t}\right) \Omega_{t} \Delta \boldsymbol{X}_{G N S S}+\boldsymbol{\xi}_{V}
$$

Ignoring a sensor displacement in the aircraft body, a barometer gives an atmospheric pressure measurement (after temperature correction) which is related to the aircraft altitude.

$$
z_{P}=P+\xi_{P}=P_{0_{t}} e^{\frac{g M}{R_{0} T_{0}}\left(Z_{t}-h_{0}\right)}+\xi_{P}
$$

where $h_{0}$ is the MSL height of the NED frame origin. $M, R_{0}$ and $T_{0}$ are the known constants of the standard atmosphere. 
For the position information, the stereo camera system outputs pixel-coordinates of the threshold center point $\boldsymbol{p}_{T H D}$ (Fig. 7) and its stereo disparity $d_{T H D}$.

$$
\boldsymbol{z}_{T H D}=\left[\begin{array}{c}
\boldsymbol{p}_{T H D} \\
d_{T H D}
\end{array}\right]+\boldsymbol{\xi}_{T H D k}=\left[\begin{array}{c}
\boldsymbol{p}\left(\boldsymbol{X}_{T H D_{t}}^{C}\right) \\
\frac{f_{x} B}{X_{T H D_{t}}^{C}}
\end{array}\right]+\boldsymbol{\xi}_{T H D k}
$$

where $f_{x}$ is a focal length found in the camera matrix $\mathcal{C}$ in (1), $B$ is a stereo baseline, and

$$
\boldsymbol{X}_{T H D_{t}}^{C}=R\left(\boldsymbol{q}_{C / B}^{*}\right)\left(R\left(\boldsymbol{q}_{t}^{*}\right)\left(\boldsymbol{X}_{T H D}-\boldsymbol{X}_{t}\right)-\Delta \boldsymbol{X}_{C A M}\right)
$$

is the threshold center point position in the camera frame.

4) Delayed ESKF with image-trigger signals: The Error State Kalman Filter (ESKF) is applied to each filter design. The idea of ESKF is to apply a linear Kalman filtering process to estimate only the error state $\boldsymbol{\delta} \boldsymbol{x}$, and then inject this estimate $\boldsymbol{\delta} \hat{\boldsymbol{x}}$ into the nominal state $\boldsymbol{x}$ for obtaining the estimated true state $\hat{\boldsymbol{x}}_{t}=\boldsymbol{x} \oplus \boldsymbol{\delta} \hat{\boldsymbol{x}}$. After each correction, the nominal state and the error state estimate are reset to $\hat{\boldsymbol{x}}_{t}$ and zero respectively, and so is the error covariance to $G^{\oplus} P G^{\oplus}$ with a state injection matrix $G^{\oplus}$ derived from the $\oplus$ operation function (2) or (4). This reset keeps the error state always around zero, and hence a linearization validity of the error state kinematics. Fig. 8 shows the ESKF scheme.

Both the attitude and position estimations rely on the stereo camera measurements which arrive with a nonnegligible delay due to image processing time. In our case, this delay is about 105 (msec) for 15 FPS (i.e. the delay is longer than one frame). Our design benefits from the image-trigger signals for incorporating the delayed vision measurement in the ESKF framework in an efficient manner.

Let $k_{i}$ be a time step at which KF correction is performed with the $i$-th measurement $\boldsymbol{z}_{i}$, and $m_{i}\left(\leq k_{i}\right)$ be a time step when that measurement was taken (Fig.9). In our example, an image is triggered at $t_{m_{i}}$ and the runway feature positions detected on that image are delivered at $t_{k_{i}}$. Starting from the current predicted state $\boldsymbol{\delta} \hat{\boldsymbol{x}}_{k_{i}}^{-}(=\mathbf{0})$ and its error covariance $P_{k_{i}}^{-}$at $t_{k_{i}}$, the delayed KF process[6] first backpropagates the state estimate to the time $t_{m_{i}}$ for calculating the measurement residual, and makes a direct correction on the current state prediction. Let $\hat{\boldsymbol{x}}_{m_{i}}^{+}=\boldsymbol{x}_{m_{i}} \oplus \boldsymbol{\delta} \hat{\boldsymbol{x}}_{m_{i}}^{+}$be

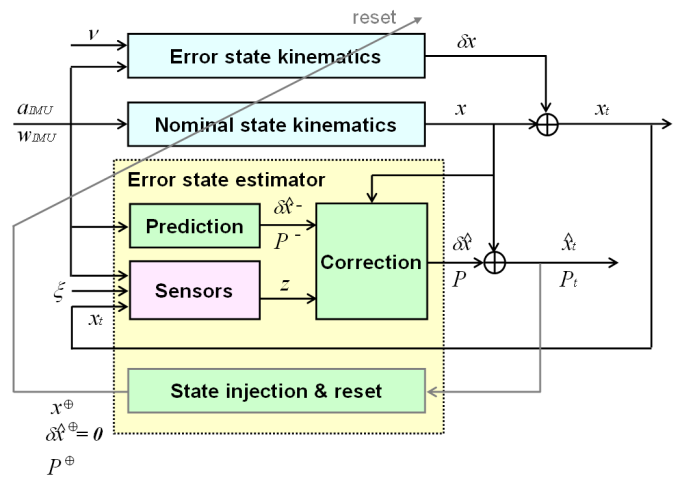

Fig. 8. Error State Kalman Filter Scheme

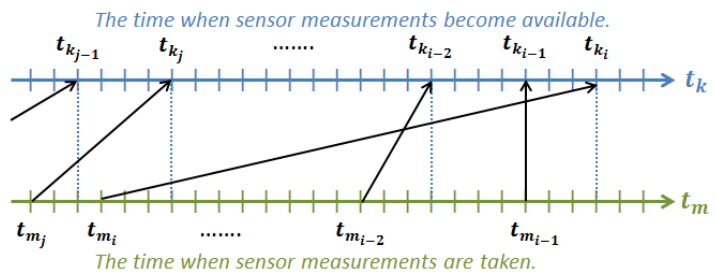

Fig. 9. Timelines for delayed sensor measurements

the back-propagated state estimate. Then the measurement residual can be obtained as follows.

$$
\begin{aligned}
\Delta \boldsymbol{z}_{i} & =\boldsymbol{z}_{i}-\boldsymbol{h}_{i}\left(\hat{\boldsymbol{x}}_{m_{i}}^{+}\right) \\
& =\boldsymbol{h}_{i}\left(\boldsymbol{x}_{m_{i}} \oplus \boldsymbol{\delta} \boldsymbol{x}_{m_{i}}\right)+\overline{\boldsymbol{\xi}}_{i}-\boldsymbol{h}_{i}\left(\boldsymbol{x}_{m_{i}} \oplus \boldsymbol{\delta} \hat{\boldsymbol{x}}_{m_{i}}^{+}\right) \\
& =\left.\frac{\partial \boldsymbol{h}_{i}(\boldsymbol{x} \oplus \boldsymbol{\delta} \boldsymbol{x})}{\partial \boldsymbol{\delta} \boldsymbol{x}}\right|_{\substack{\boldsymbol{x}=\boldsymbol{x}_{m_{i}} \\
\boldsymbol{\delta} \boldsymbol{x}=\hat{\boldsymbol{x}}_{m_{i}}^{+}}}\left(\boldsymbol{\delta} \boldsymbol{x}_{m_{i}}-\boldsymbol{\delta} \hat{\boldsymbol{x}}_{m_{i}}^{+}\right)+\overline{\boldsymbol{\xi}}_{i} \\
& =H_{i} \boldsymbol{\delta} \tilde{\boldsymbol{x}}_{m_{i}}^{+}+\overline{\boldsymbol{\xi}}_{i}
\end{aligned}
$$

where $\boldsymbol{h}_{i}\left(\boldsymbol{x}_{t}\right)$ is a nonlinear sensor model of the $i$-th measurement (introduced for each sensor in the two previous subsections). $\overline{\boldsymbol{\xi}}_{i}$ is an augmented measurement noise including the original sensor error $\boldsymbol{\xi}_{i}$, the IMU sensor noise, and the attitude estimation error. Then the KF correction is performed with (6) as below.

$$
\left\{\begin{array}{l}
\boldsymbol{\delta} \hat{\boldsymbol{x}}_{k_{i}}=\boldsymbol{\delta} \hat{\boldsymbol{x}}_{k_{i}}^{-}+K_{i} \Delta \boldsymbol{z}_{i}=K_{i} \Delta \boldsymbol{z}_{i} \\
P_{k_{i}}=P_{k_{i}}^{-}-K_{i} H_{i} P_{m_{i} k_{i}} \\
K_{i}=P_{m_{i} k_{i}}^{T} H_{i}^{T}\left(H_{i} P_{m_{i}}^{+} H_{i}^{T}+\bar{R}_{i}\right)^{-1}
\end{array}\right.
$$

where $P_{m_{i}}^{+}=\mathbb{E}\left[\boldsymbol{\delta} \tilde{\boldsymbol{x}}_{m_{i}}^{+} \boldsymbol{\delta} \tilde{\boldsymbol{x}}_{m_{i}}^{+}{ }^{T}\right]$ is an error covariance of the back-propagated estimate and $P_{m_{i} k_{i}}=\mathbb{E}\left[\boldsymbol{\delta} \tilde{\boldsymbol{x}}_{m_{i}}^{+} \boldsymbol{\delta} \tilde{\boldsymbol{x}}_{k_{i}}^{-T}\right]$ is its correlation with the current prediction error.

The idea of the delayed ESKF in this work is to use the image trigger signal to notify the filter of the time instant $t_{m_{i}}$ without delay, so that it can run the back-propagation (BP) process forward in time to obtain $\boldsymbol{\delta} \hat{\boldsymbol{x}}_{m_{i}}^{+}, P_{m_{i}}^{+}$and $P_{m_{i} k_{i}}$. Then when the image processing output arrives at $t_{k_{i}}\left(>t_{m_{i}}\right)$, the filter will be already ready to do (7).

Upon a reception of the image trigger signal at $t_{m_{i}}$, the $\mathrm{BP}$ process is initialized with

$$
\boldsymbol{\delta} \hat{\boldsymbol{x}}_{m_{i}}^{+}=\mathbf{0}, \quad \Gamma_{i}=I, \quad \Delta Q_{i}=O, \quad \delta Q_{i}=O
$$

At each ESKF prediction at $t_{\ell}$, the above matrices are propagated with a state transition matrix $\Phi_{\ell}$ and a covariance $\bar{Q}_{\ell}$ of the augmented process noise (given in (3) or (5)).

$$
\Gamma_{i}=\Phi_{\ell} \Gamma_{i}, \quad \Delta Q_{i}=\Phi_{\ell} \Delta Q_{i} \Phi_{\ell}^{T}+\bar{Q}_{\ell}, \quad \delta Q_{i}=\Phi_{\ell} \delta Q_{i} \Phi_{\ell}^{T}
$$

At each ESKF correction performed at $t_{k_{j}}(j<i)$, the BP states are also updated with the correction term as below.

$$
\left\{\begin{array}{l}
\boldsymbol{\delta} \hat{\boldsymbol{x}}_{m_{i}}^{+}=\boldsymbol{\delta} \hat{\boldsymbol{x}}_{m_{i}}^{+}+\Gamma_{i}^{-1} K_{j} \Delta \boldsymbol{z}_{j} \\
\delta Q_{i}=\left(I-K_{j} \bar{H}_{j}\right) \delta Q_{i}+\gamma K_{j} \bar{H}_{j} \Delta Q_{i}
\end{array}\right.
$$

where $\bar{H}_{j}=H_{j} \Gamma_{j}^{-1}, \gamma=0$ when the $j$-th measurement is the delayed stereo camera measurement on the previous image frame (recalling that the delay is longer than one 
frame) and 1 for the other non-delayed measurements. Then, upon the ESKF reset after each correction at $t_{k_{j}}$, the BP matrices are also reset to

$$
\Gamma_{i}=G_{j}^{\oplus} \Gamma_{i}, \quad \Delta Q_{i}=G_{j}^{\oplus} \Delta Q_{i} G_{j}^{\oplus^{T}}, \quad \delta Q_{i}=G_{j}^{\oplus} \delta Q_{i} G_{j}^{\oplus^{T}}
$$

with the state injection matrix. We continue these BP operations until the corresponding vision measurement arrives at $t_{k_{i}}$, when (6) and (7) are done with the resulting $\boldsymbol{\delta} \hat{\boldsymbol{x}}_{m_{i}}^{+}$and

$$
\begin{aligned}
P_{m_{i}}^{+} & =\Gamma_{i}^{-1}\left(P_{k_{i}}^{-}-\Delta Q_{i}+\delta Q_{i}+\delta Q_{i}^{T}\right) \Gamma_{i}^{-T} \\
P_{m_{i} k_{i}} & =\Gamma_{i}^{-1}\left(P_{k_{i}}^{-}-\Delta Q_{i}+\delta Q_{i}^{T}\right)
\end{aligned}
$$

The detailed derivation is provided in [7].

\section{B. Integrity monitoring}

An integrity monitoring (IM) function is added to the navigation filter for the purpose of sensor fault detection and Protection Level (PL) calculation, which is a requirement for an aircraft navigation system in civil aviation. It applies a well-developed residual-based LS-RAIM algorithm to a batch-realization of the proposed delayed ESKF framework, similarly as done in [8] for the normal KF.

1) Batch realization of the delayed ESKF: Consider that the filter is initialized at a time step $k_{0}$ with $\boldsymbol{x}_{k_{0}}, \boldsymbol{\delta} \hat{\boldsymbol{x}}_{k_{0}}=$ $\mathbf{0}$ and $P_{k_{0}}$. The time-delayed ESKF processes from $k_{0}$ to a time step $k$ can be re-written in a batch realization form with $\boldsymbol{v}_{K} \sim \mathcal{N}\left(\mathbf{0}, V_{K}\right)$ and a bias fault vector $\boldsymbol{f}_{K}$.

$$
\boldsymbol{\delta} \boldsymbol{z}_{K}=\mathcal{H}_{K} \boldsymbol{\delta} \boldsymbol{x}_{K}+\boldsymbol{v}_{K}+\mathcal{T}_{K} \boldsymbol{f}_{K}
$$

At time $k_{0}$, the batch realization form is initialized with $\boldsymbol{\delta} \boldsymbol{z}_{K_{0}}=\mathbf{0}, \mathcal{H}_{K_{0}}=\mathcal{T}_{K_{0}}=I, V_{K_{0}}=P_{k_{0}}$ and unknown

$\boldsymbol{\delta} \boldsymbol{x}_{K_{0}}=\boldsymbol{\delta} \boldsymbol{x}_{k_{0}}, \quad \boldsymbol{v}_{K_{0}}=\mathbb{E}\left[\boldsymbol{\delta} \boldsymbol{x}_{k_{0}}\right]-\boldsymbol{\delta} \boldsymbol{x}_{k_{0}}, \quad \boldsymbol{f}_{K_{0}}=-\mathbb{E}\left[\boldsymbol{\delta} \boldsymbol{x}_{k_{0}}\right]$

$\boldsymbol{f}_{K_{0}}$ corresponds to the initial estimation bias (which may be due to sensor fault occurred before $k_{0}$ ). Then (9) is incrementally defined at each time step. When the KF prediction is performed at time $k+1$,

$$
\begin{aligned}
& \boldsymbol{\delta}_{K+1}=\left[\begin{array}{cc}
\boldsymbol{\delta} \boldsymbol{z}_{K}^{T} & \left(\bar{\Phi}_{k+1} \boldsymbol{\delta} \hat{\boldsymbol{x}}_{k}\right)^{T}
\end{array}\right]^{T} \\
& =\left[\begin{array}{cc}
\mathcal{H}_{K} & O \\
{\left[\begin{array}{cc}
O & \bar{\Phi}_{k+1}
\end{array}\right]} & -I
\end{array}\right]\left[\begin{array}{c}
\boldsymbol{\delta} \boldsymbol{x}_{K} \\
\boldsymbol{\delta} \boldsymbol{x}_{k+1}
\end{array}\right]+\left[\begin{array}{c}
\boldsymbol{v}_{K} \\
\overline{\boldsymbol{\nu}}_{k+1}
\end{array}\right]+\left[\begin{array}{c}
\mathcal{T}_{K} \\
O
\end{array}\right] \boldsymbol{f}_{K} \\
& =\mathcal{H}_{K+1} \boldsymbol{\delta} \boldsymbol{x}_{K+1}+\boldsymbol{v}_{K+1}+\mathcal{T}_{K+1} \boldsymbol{f}_{K+1}
\end{aligned}
$$

with $V_{K+1}=\operatorname{blkdiag}\left(V_{K}, \bar{Q}_{k+1}\right)$. When there is no correction at the previous time step $k, \bar{\Phi}_{k+1}=\Phi_{k+1}$ is a normal state transition matrix and $\boldsymbol{\delta} \hat{\boldsymbol{x}}_{k}=\mathbf{0}$. Otherwise, letting $k=k_{i-1}, \bar{\Phi}_{k+1}=\Phi_{k+1} G_{i-1}^{\oplus}$ and $\boldsymbol{\delta} \hat{\boldsymbol{x}}_{k}=K_{i-1} \Delta \boldsymbol{z}_{i-1}$ from (7). When the KF correction is performed at $k+1=k_{i}$ after the prediction, (10) is further updated to

$$
\begin{aligned}
& \boldsymbol{\delta} \boldsymbol{z}_{K+1}=\left[\begin{array}{ll}
\boldsymbol{z}_{K+1}^{T} & \left(\Delta \boldsymbol{z}_{i}+H_{i} \boldsymbol{\delta} \hat{\boldsymbol{x}}_{m_{i}}^{+}\right)^{T}
\end{array}\right] \\
= & {\left[\begin{array}{c}
\mathcal{H}_{K+1} \\
H_{i} E_{m_{i}}^{T}
\end{array}\right] \boldsymbol{\delta} \boldsymbol{x}_{K+1}+\left[\begin{array}{c}
\boldsymbol{v}_{K+1} \\
\overline{\boldsymbol{\xi}}_{i}
\end{array}\right]+\left[\begin{array}{cc}
\mathcal{T}_{K+1} & O \\
O & I
\end{array}\right]\left[\begin{array}{c}
\boldsymbol{f}_{K+1} \\
\boldsymbol{f}_{k_{i}}
\end{array}\right] } \\
= & \mathcal{H}_{K+1} \boldsymbol{\delta} \boldsymbol{x}_{K+1}+\boldsymbol{v}_{K+1}+\mathcal{T}_{K+1} \boldsymbol{f}_{K+1}
\end{aligned}
$$

with $V_{K+1}=\operatorname{blkdiag}\left(V_{K+1}, \bar{R}_{i}\right) . E_{m_{i}}^{T}$ is a matrix to choose the error state vector at a time step $m_{i}$ among $\boldsymbol{\delta} \boldsymbol{x}_{K+1} \cdot \boldsymbol{f}_{k_{i}}$ is a bias fault on the sensor measurement obtained at $k_{i}$.
2) Application of LS-RAIM algorithm: This paper applies the residual-based LS-RAIM algorithm to (9) for integrity monitoring. The LS-estimate of $\boldsymbol{\delta} \boldsymbol{x}_{K}$ is given by

$$
\boldsymbol{\delta} \hat{\boldsymbol{x}}_{K \mid K}=S_{K} \boldsymbol{\delta} \boldsymbol{z}_{K}=\boldsymbol{\delta} \boldsymbol{x}_{K}+S_{K}\left(\boldsymbol{v}_{K}+\mathcal{T}_{K} \boldsymbol{f}_{K}\right)
$$

where $\mathcal{P}_{K \mid K}=\left(\mathcal{H}_{K}^{T} V_{K}^{-1} \mathcal{H}_{K}\right)^{-1}$ and $S_{K}=\mathcal{P}_{K \mid K} \mathcal{H}_{K}^{T} V_{K}^{-1}$. Its error $\boldsymbol{\delta} \tilde{\boldsymbol{x}}_{K \mid K}$ follows $\mathcal{N}\left(-S_{K} \mathcal{T}_{K} \boldsymbol{f}_{K}, \mathcal{P}_{K \mid K}\right)$. The LSestimate of the current state is equivalent to the ESKF estimate before injection: $\boldsymbol{\delta} \hat{\boldsymbol{x}}_{k \mid K}=\boldsymbol{\delta} \hat{\boldsymbol{x}}_{k}, \mathcal{P}_{k \mid K}=P_{k}$.

a) Fault detection: From the residual vector $\boldsymbol{r}_{K \mid K}=$ $\boldsymbol{\delta} \boldsymbol{z}_{K}-\mathcal{H}_{K} \boldsymbol{\delta} \hat{\boldsymbol{x}}_{K \mid K}$, a residual-based fault detector can be defined by

$$
q_{K}^{2}=\boldsymbol{r}_{K \mid K}^{T} V_{K}^{-1} \boldsymbol{r}_{K \mid K} \sim \chi^{2}\left(m_{K}-n_{K}, \lambda_{K}^{2}\right)
$$

which follows a $\chi^{2}$-distribution with non-centrality $\lambda_{K}^{2}=$ $\boldsymbol{f}_{K}^{T} \mathcal{T}_{K}^{T} V_{K}^{-1}\left(I-\mathcal{H}_{K} S_{K}\right) \mathcal{T}_{K} \boldsymbol{f}_{K} \cdot m_{K}$ and $n_{K}$ are dimensions of $\boldsymbol{\delta} \boldsymbol{x}_{K}$ and $\boldsymbol{\delta} \boldsymbol{z}_{K}$ respectively. The detection threshold $q_{T_{K}}^{2}$ can be determined by limiting the false alarm probability (under fault-free hypothesis $F_{0}$ where $\boldsymbol{f}_{K}=\mathbf{0}$ ) by a given continuity risk requirement $P_{C R}$.

$$
\operatorname{Pr}\left(q_{K}^{2} \geq q_{T_{K}}^{2} \mid F_{0}\right) \operatorname{Pr}\left(F_{0}\right) \leq \operatorname{Pr}\left(q_{K}^{2} \geq q_{T_{K}}^{2} \mid F_{0}\right)=P_{C R}
$$

b) Integrity risk requirement: Let $\boldsymbol{y}_{k}=A^{T} \boldsymbol{x}_{t_{k}}=$ $A^{T}\left(\boldsymbol{x}_{k} \oplus \boldsymbol{\delta} \boldsymbol{x}_{k}\right)$ be a current state-of-interest, for which integrity should be monitored. It could be a horizontal position, altitude or any other states. Its LS-estimation error follows $\tilde{\boldsymbol{y}}_{k \mid K} \sim \mathcal{N}\left(-A_{K}^{T} S_{K} \mathcal{T}_{K} \boldsymbol{f}_{K}, A_{k}^{T} \mathcal{P}_{k \mid K} A_{k}\right)$ where

$$
A_{K}^{T}=A_{k}^{T} E_{k}^{T}=\left.A^{T} \frac{\partial(\boldsymbol{x} \oplus \boldsymbol{\delta} \boldsymbol{x})}{\partial \boldsymbol{\delta} \boldsymbol{x}}\right|_{\substack{\boldsymbol{x}=\boldsymbol{x}_{k} \\ \boldsymbol{\delta}=\boldsymbol{\delta} \hat{\boldsymbol{x}}_{k}}} E_{k}^{T}
$$

The integrity risk under each fault mode $F_{n}$ is defined as

$$
P_{I_{K}}\left(F_{n}\right)=\operatorname{Pr}\left(\left\|\tilde{\boldsymbol{y}}_{k \mid K}\right\|>A L \mid F_{n}\right) \operatorname{Pr}\left(q_{K}^{2}<q_{T_{K}}^{2} \mid F_{n}\right)
$$

for a given Alert Limit $A L$. It is a probability of having the estimation error beyond $A L$ without detecting it. The integrity risk requirement is satisfied if $P_{I_{K}}\left(F_{n}\right) \leq P_{I R_{n}}$ for all the fault modes $F_{n}$, where $P_{I R_{n}}$ is determined from a given total integrity risk requirement $P_{I R}$ and risk allocation.

c) Worst-case fault: The worst-case fault $\boldsymbol{f}_{n}^{*} \in F_{n}$ is defined as a fault maximizing its integrity risk (14). Let $C_{K}^{n}=\operatorname{blkdiag}\left(C_{0}^{n}, \cdots, C_{i}^{n}\right)$ be a faulty sensor selection matrix of the fault mode $F_{n}$ at time step $k$, which extracts only the non-zero elements $\boldsymbol{f}_{N Z_{n}}$ of the fault vector $\boldsymbol{f}_{n}$, i.e., $\boldsymbol{f}_{n}=C_{K}^{n} \boldsymbol{f}_{N Z_{n}}$. For example, we can imagine a fault mode, where the GNSS pseudo-range measurements of one specific satellite is biased. Then the worst-case fault $f_{n}^{*}$ at time $k$ is the one maximizing the failure mode slope:

$$
g_{K}^{2}\left(\boldsymbol{f}_{n}\right)=\frac{\boldsymbol{f}_{N Z_{n}}^{T} \mathcal{T}_{K}^{n T} S_{K}^{T} A_{K} A_{K}^{T} S_{K} \mathcal{T}_{K}^{n} \boldsymbol{f}_{N Z_{n}}}{\boldsymbol{f}_{N Z_{n}}^{T} \mathcal{T}_{K}^{n T} V_{K}^{-1}\left(I-\mathcal{H}_{K} S_{K}\right) \mathcal{T}_{K}^{n} \boldsymbol{f}_{N Z_{n}}}
$$

where $\mathcal{T}_{K}^{n}=\mathcal{T}_{K} C_{K}^{n}$. When the full state is still observable only with the non-faulty measurements, $\mathcal{T}_{K}^{n T} V_{K}^{-1}(I-$ $\left.\mathcal{H}_{K} S_{K}\right) \mathcal{T}_{K}^{n}$ is invertible and let us denote its inverse by $M_{K}^{n}$. Then the failure mode slope (15) is upper-bounded by the maximum eigenvalue $\lambda_{\max }$ of a matrix $A_{k}^{T} \tilde{M}_{k}^{n} A_{k}=$ 
$A_{K}^{T} S_{K} \mathcal{T}_{K}^{n} M_{K}^{n} \mathcal{T}_{K}^{n T} S_{K}^{T} A_{K}$. Then the worst-case fault $\boldsymbol{f}_{n}^{*}$ can be determined as below.

$$
\boldsymbol{f}_{n}^{*}=\frac{f_{n}^{*}}{\sqrt{\lambda_{\max }}} C_{K}^{n} M_{K}^{n} \mathcal{T}_{K}^{n T} S_{K}^{T} A_{K} \boldsymbol{v}_{\max }
$$

where $\boldsymbol{v}_{\text {max }}$ is a unit eigenvector of $A_{k}^{T} \tilde{M}_{k}^{n} A_{k}$ corresponding to $\lambda_{\max }$. When $\boldsymbol{f}_{K}=\boldsymbol{f}_{n}^{*}$, the probability distributions of the fault detector $q_{K}^{2}$ and the estimation error $\tilde{\boldsymbol{y}}_{k \mid K}$ become

$$
\left\{\begin{array}{l}
q_{K}^{2}\left(\boldsymbol{f}_{n}^{*}\right) \sim \chi^{2}\left(m_{K}-n_{K}, f_{n}^{* 2}\right) \\
\tilde{\boldsymbol{y}}_{k \mid K}\left(\boldsymbol{f}_{n}^{*}\right) \sim \mathcal{N}\left(-f_{n}^{*} \sqrt{\lambda_{\max }} \boldsymbol{v}_{\max }, A_{k}^{T} P_{k \mid K} A_{k}\right)
\end{array}\right.
$$

The worst-case fault magnitude $f_{n}^{*}$, which maximizes the integrity risk (14) under (17), can be found by a simple line search algorithm. Since $P_{I_{K}}\left(\boldsymbol{f}_{n}^{*}\right)$ is an upper limit of (14), the integrity risk requirement is conservatively guaranteed if, for all the fault modes $F_{n}, P_{I_{K}}\left(f_{n}^{*}\right) \leq P_{I R_{n}}$. See [12] for details of the worst-case fault derivation.

d) Protection Level: Protection Level (PL) is defined by the minimum $A L$ respecting the integrity risk requirement. That is, when no detection, the estimation error is guaranteed to lie within the PL-bound for a probability of $\left(1-P_{I R}\right)$. For each fault mode $F_{n}$, the PL can be conservatively approximated by a solution for $A L$ of $P_{I_{K}}\left(\boldsymbol{f}_{n}^{*}\right)=P_{I R_{n}}$. By applying the triangle inequality to $\left\|\tilde{\boldsymbol{y}}_{k \mid K}\right\|$, the PL can be further approximated as follows.

$$
P L\left(F_{n}\right)=P L_{n}=\sigma_{n}+\left|f_{n}^{*}\right| \sqrt{\lambda_{\max }}
$$

where, for $\Delta \tilde{\boldsymbol{y}}_{k \mid K} \sim \mathcal{N}\left(\mathbf{0}, A_{k}^{T} \mathcal{P}_{k \mid K} A_{k}\right), \sigma_{n}$ satisfies

$$
\operatorname{Pr}\left(\left\|\Delta \tilde{\boldsymbol{y}}_{k \mid K}\right\| \leq \sigma_{n}\right)=1-\frac{P_{I R_{n}}}{\operatorname{Pr}\left(q_{K}^{2}<q_{T_{K}}^{2} \mid \boldsymbol{f}_{n}^{*}\right)}
$$

For the fault-free mode $F_{0}$, we have $\boldsymbol{f}_{0}^{*}=\mathbf{0}$ and hence $P L_{0}=\sigma_{0}$ with $\operatorname{Pr}\left(q_{K}^{2}<q_{T_{K}}^{2} \mid F_{0}\right)=1-P_{C R}$. The global PL can be given by the maximum among all the fault modes:

$$
P L_{K}=\max _{F_{n} \in \mathcal{F}_{K}} P L\left(F_{n}\right)
$$

3) Implementation: Only the detector value $q_{K}^{2}$ in (12), the degree-of-freedom $m_{K}-n_{K}$ of its $\chi^{2}$-distribution, and the matrix $\tilde{M}_{k}^{n}$ for each fault mode $F_{n}$ are necessary for obtaining the probability distributions (17). These can be computed recursively from the delayed ESKF process. It should be noted that there is no need to calculate the worstcase fault vector itself (16), and hence nor to track $M_{K}^{n}$.

The fault detector and the degree-of-freedom are set to zero at the initial time step $k_{0}$. Their values are incremented upon each ESKF correction (7) at time step $k+1=k_{i}$ :

$$
\left\{\begin{array}{l}
\left(m_{K+1}-n_{K+1}\right)=\left(m_{K}-n_{K}\right)+m_{i} \\
q_{K+1}^{2}=q_{K}^{2}+\Delta \boldsymbol{z}_{i}^{T}\left(H_{i} P_{m_{i}}^{+} H_{i}^{T}+\bar{R}_{i}\right)^{-1} \Delta \boldsymbol{z}_{i}
\end{array}\right.
$$

where $m_{i}$ is a dimension of the $i$-th sensor's measurement. The matrix $\tilde{M}_{k}^{n}$ can be given by

$$
\tilde{M}_{k}^{n}=E_{k}^{T}\left(\mathcal{M}_{K \mid K}^{n}-\mathcal{P}_{K \mid K}\right) E_{k}=\mathcal{M}_{k \mid K}^{n}-\mathcal{P}_{k \mid K}
$$

where $\mathcal{P}_{k \mid K}=P_{k}$ from the ESKF result. The matrix $\mathcal{M}_{K \mid K}^{n}$ corresponds to the error covariance of the LS-estimate only with the non-faulty measurements under $F_{n}$. In consequence, $\mathcal{M}_{k \mid K}^{n}$ is the error covariance of the sub-set measurement ESKF, and the matrix $\tilde{M}_{k}^{n}$ is equivalent to its difference from that of the full-set ESKF, like in another RAIM algorithm based on Solution Separation[12].

4) Reset mechanism: As time step proceeds, the detector value and the degree-of-freedom keep increasing by (20). The fault mode set also augments considerably, which makes the IM process computationally heavy since it runs a bank of the sub-set ESKF filters in parallel for all the fault modes. Hence we introduce a reset mechanism to re-initialize the IM process regularly. Since an IM process is valid only when the full-state is observable with the sub-set measurements for all the fault modes, several IM processes are run over different time windows so that at least one of them is valid. A key idea of our reset mechanism is that all the ESKF filters run in an IM process are initialized without relying on the estimation result of the main navigation filter. This is equivalent to supposing $\boldsymbol{f}_{K_{0}} \neq \mathbf{0}$ under all the fault hypotheses. By doing so, we can remove an influence (bias) of past undetected faults on the newly reset detector, and ensure an existence of at least one non-faulty sub-set filter in the IM process.

\section{SYSTEM IMPLEMENTATION}

\section{A. K50-Advanced UAV platform and flight avionics system}

An experimental UAV platform used in this work is K50-Advanced fixed-wing aircraft (Fig.1) manufactured by a Spanish SME, USOL, within the VISION project. Its specification is given with the figure. The K50 aircraft design features its high payload capacity $(100 \mathrm{~L}, 20 \mathrm{~kg}$ ), which is very important for the experimental usage.

The main navigation sensors used in the flight avionics, as well as in evaluation of this work, are listed in Tab.II. The avionics system has been fully developed by ONERA from electronics design to flight control software. Its hardware consists of three micro-controllers of Cortex M4 and data recorder of $1 \mathrm{~GB}$ (about 3 hours of flight). It is a redundant system with four electronics cards (Fig.10): RC card for Primary control link, Connector card for connections to the onboard sensors and actuators, GNC card for auto-pilot functions, and Safety card for replacing the GNC card in case of its failure. The flight control software covers the three different user cases of Manual, Automatic and Fail-safe flight modes. It also allows an external system (payload) to intervene at any level of the GNC function hierarchy (actuator inputs, attitude commands, velocity/altitude commands, waypoints, etc.).

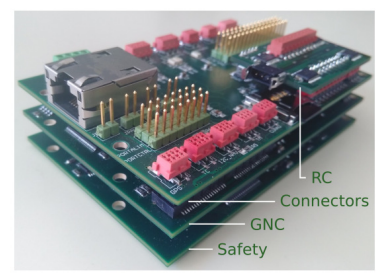

Fig. 10. ONERA flight avionics hardware 
TABLE II

NAVIGATION SENSORS ONBOARD THE K50

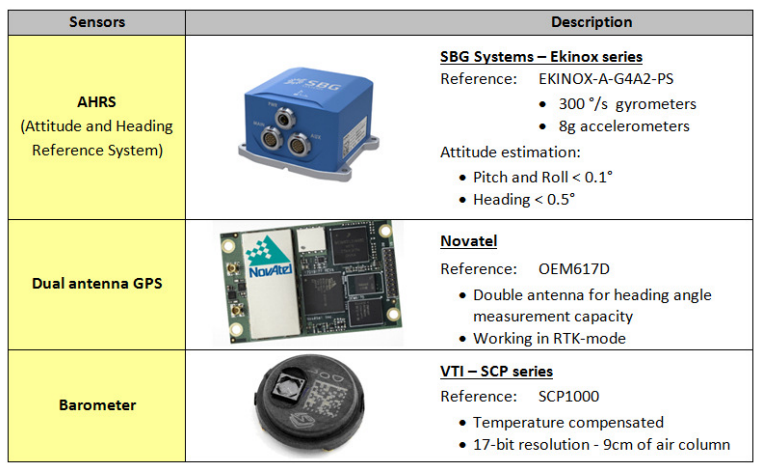

\section{B. Payload integration}

On the top of the flight avionics, ONERA's payload (PL) computer is integrated on the K50. It serves as an interface of different external systems (notably the RICOH stereo camera system) with the flight avionics, for hosting the experimental GNC algorithms, as well as for the PL communications with Ground Control Station. The embedded computer is composed of Conga-TC87 module with Intel Core i7-4650U dual core processors $(2 \times 1.7 / 3.3 \mathrm{GHz}, 4 \mathrm{MB}$ Cache, $15 \mathrm{~W}), 64$ GB hard drive and 8 GB RAM. It is managed by a custom Ubuntu server 14.04 with real-time features.

The PL software architecture is based on ROS Indigo (Robotic Operating System - http://www.ros.org/) and a custom component-based framework called MAUVE [13]. The navigation system proposed in this paper is realized within this architecture by creating different independent components (=processes) as illustrated in ellipses in Fig. 11. Each component executes a specific function. For example, the delayed ESKF filter process presented in Section III is implemented in the 'Estimator' component. 'RICOH UART' component is a RS232C driver to read the image processing output data and the image trigger timestamp sent from the external RICOH payload computer.

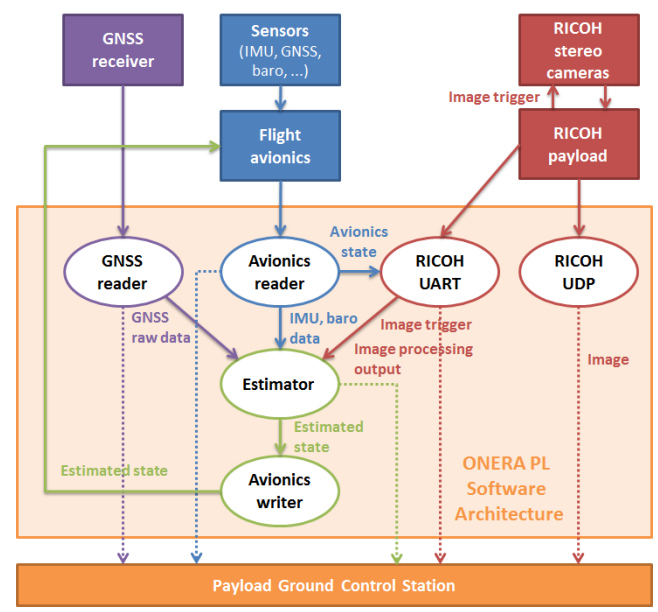

Fig. 11. Software architecture realization on the payload

\section{Time synchronization}

Time synchronization is a cornerstone of the system integration. As seen in Fig.11, the PL receives IMU and barometer measurements from the flight avionics, GNSS measurements directly from the receiver and the stereo camera measurements from the RICOH PL. These measurements are timestamped by each system, and hence it is crucial for the data fusion that clocks of all those systems are synchronized. To achieve a precise time synchronization, we have implemented a synchronization procedure based on a PPS (Pulse Per Second) signal and a RS232 frame. The PPS signal is a $0,+5 \mathrm{~V}$ signal with a frequency of $1 \mathrm{~Hz}$ and a 100 ms time-up, generated by the flight avionics system (from the GPS PPS signal). The RS232 data frame containing the avionics timestamp corresponding to a rising edge of the last PPS pulse is sent to the PL systems so that they can correct their clock offset w.r.t. the avionics' clock.

\section{EVALUATION RESULTS}

The RICOH long-range stereo camera system for runway feature detection (Section II) has been tested in real time onboard the $\mathrm{K} 50$ on the final approach trajectory. Then, the fault tolerant navigation system (Section III) has been evaluated on real sensor data sets acquired through flight experiments with GNSS failure emulations.

\section{A. Runway feature detection results}

This subsection shows results of the runway feature detection performed in a K50's flight during which five approach trajectories are made from $600(\mathrm{~m})$ away from the runway threshold. Fig.12 shows the aircraft position and attitude recovered from the stereo camera measurements for one of the final approach sequences, compared to the GPS/RTK position and the gyro attitude angles (obtained by pure integration of the gyro measurements). The stereo camera measurements well-match with the reference, proving a high precision of the runway feature detection algorithm. The runway features are detected until reaching at about 200 (m) away from the threshold.

In addition to the detection accuracy, the runway recognition and misrecognition rates are important factors that determine the performance of the stereo camera system. In this flight, there were 495 'captured' image frames in which the runway threshold marking entirely entered in the camera's capture field of view (FoV), and 426 'output' image frames for which the runway feature detection results
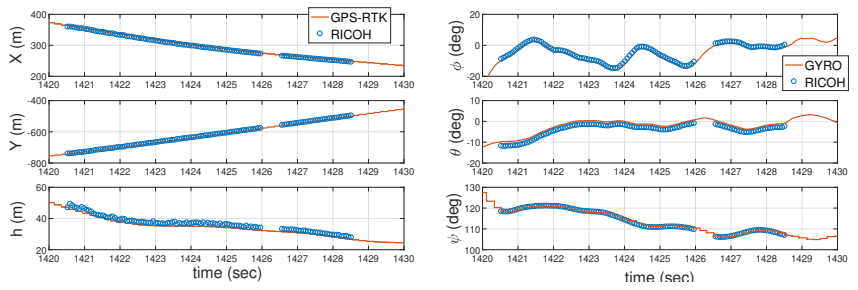

Fig. 12. The UAV position (left) and attitude (right) measurements from the stereo camera system, vs. the GPS/RTK position and the Gyro attitude 


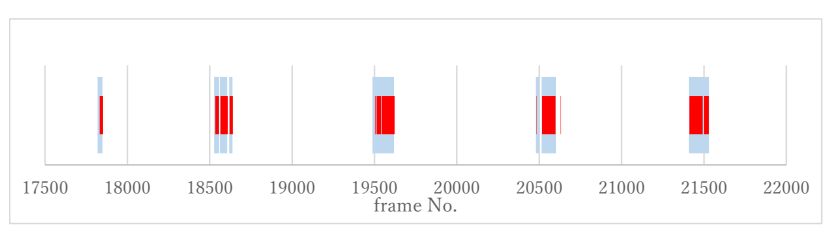

Fig. 13. Distribution of the 'captured' (blue) and 'output' (red) frames

are validated and so the navigation data is output. A ratio between these two values is $86.1 \%$. However, the 'captured' frames may include images on which the runway sidelines are beyond FoV and the navigation data calculation is not possible. There may be uncounted frames where the threshold marking is partially in FoV but the runway features were successfully detected. For this reason, this ratio does not represent a strict recognition rate but rather gives a general indication of the detection rate. Fig.13 shows the temporal distribution of the 'captured' and 'output' frames in blue and red respectively. During five approaches, aside from the cases for which the runway was outside FoV, there are two cases where the runway feature detection results were not output. The post-flight analysis found that they were due to a too-small permissible range used in the validation process of the detection results, when the UAV was at far distance from the runway. This can be improved by expanding the permissible range in function of the distance.

\section{B. Estimation and fault detection results}

1) Sensors setup: The estimation performance of the proposed navigation filter is evaluated through open-loop simulation tests with the K50 real sensor data and signals:

- IMU acceleration and angular velocity at $50 \mathrm{~Hz}$

- GPS pseudo-range for visible satellites at $20 \mathrm{~Hz}$

- GPS velocity at $20 \mathrm{~Hz}$

- Barometric pressure at $1.89 \mathrm{~Hz}$

- RICOH stereo camera at $15 \mathrm{~Hz}$ with 105 (msec) latency

- RICOH stereo camera image trigger at $15 \mathrm{~Hz}$

2) GPS signal loss: In the first test, for evaluating an effect of adding the stereo camera measurements, the GPS signal is completely cut off during a final approach phase (recalling that we are in open-loop test). Fig.14 compares the position estimation error and the estimated velocity with and without the stereo camera measurement, where the filter was initialized at $\mathrm{t}=0(\mathrm{sec})$ and a GPS signal loss (both the pseudo-ranges and velocity) was introduced at $t=2(\mathrm{sec})$. As expected, a large drift in the horizontal position estimation without GPS correction can be removed with an aide of the stereo camera.

3) GPS pseudo-range bias and Fault detection: In the second test, a GPS fault is simulated by introducing a fault percentage on the ionospheric delay correction signal on one selected satellite. It causes a bias corresponding to

$$
(1 \text { - fault percent }) \times(\text { ionospheric delay correction })
$$

in the pseudo-range measurement of that satellite. In this test, the satellite PRN 12 was chosen and its ionospheric correc-
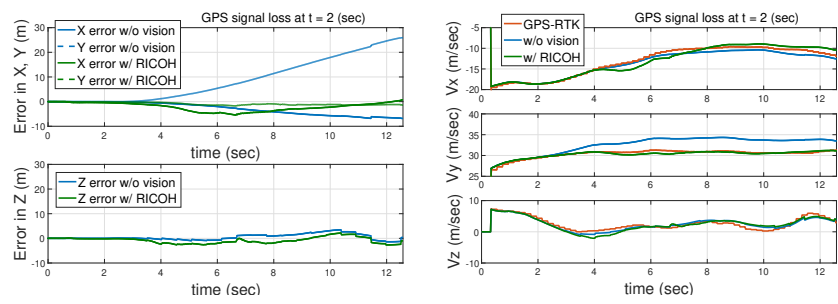

Fig. 14. The position estimation error (left) and the velocity estimation with and without the stereo camera in case of GPS signal loss

tion was about $4(\mathrm{~m})$. Step faults with different magnitude and drift (ramp) faults with different slope are emulated.

Fault detection was performed by the IM function presented in Section III-B. Considering the full-state observability under single satellite fault hypotheses, we chose to run three IM processes in parallel with the time window of 3 times barometer sampling time $(\sim 1.6 \mathrm{sec})$. The IM process is reset upon each barometer measurement reception. The IM function monitors the integrity of horizontal $(\mathrm{H})$ and vertical (V) positions, and the parameters used in this paper are: $H A L=40(\mathrm{~m}), V A L=20(\mathrm{~m}), P_{C R}=1.3333 \times 10^{-7}(/ \mathrm{h})$, $P_{I R}=4.8 \times 10^{-6}(/ \mathrm{h})$, and one satellite failure probability $P\left(F_{n}\right)=1.16 \times 10^{-5}(/ \mathrm{h})$. They correspond to the APV-II aircraft approach requirements in civil aviation[14].

Fig.15 shows the results of Time-to-Alert (TTA) of the IM function after an occurrence of the step/drift bias fault. TTAs are plotted versus fault magnitude/slope, comparing between the navigation systems with (in green) and without (in blue) the stereo camera measurements. In both figures, the green plots locate lower and more on the left than the blue ones. It implies the improvement in fault detectability, being capable of detecting smaller faults in shorter time. The dashed lines are the results when using also the GPS velocity measurements. Surprisingly, this non-faulty velocity information did not improve the detection time of step bias fault. This might be due to that the GPS velocity error covariance used in the navigation filter was much larger than it should be.

Fig. 16 and 17 show time profiles of the fault detector value and its detection threshold, calculated by the main full-set navigation filter (left) and by the sub-set measurement filters for each single satellite fault mode (right). Fig.16 is for the $70 \%$ step fault percentage, and Fig. 17 is for the 20\%/sec drift fault. These are the results of the navigation system using the
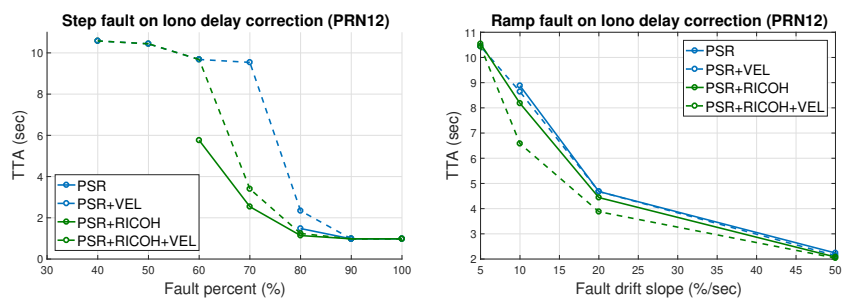

Fig. 15. Time-to-Alert results for Step (left) and Drift (right) bias faults with and without the stereo camera system 

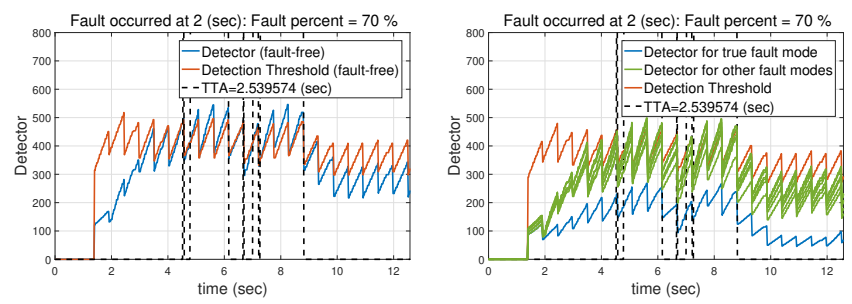

Fig. 16. Fault detector value and its detection threshold when introducing $70 \%$ step fault percentage at $\mathrm{t}=2(\mathrm{sec})$
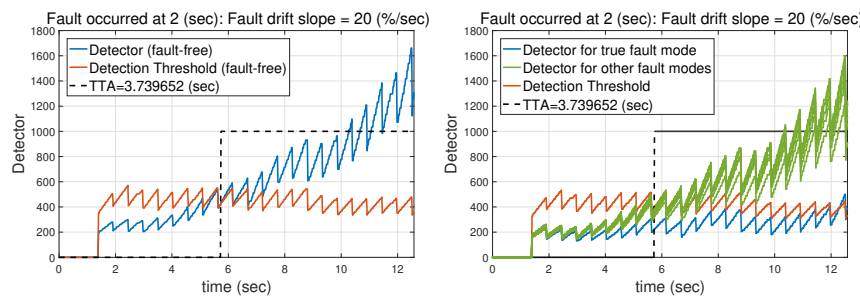

Fig. 17. Fault detector value and its detection threshold when introducing $20 \% / \mathrm{sec}$ drift fault percentage at $\mathrm{t}=2(\mathrm{sec})$

stereo camera but not the GPS velocity measurements. TTA corresponds to the time at which the full-set filter detector value (blue) exceeds its threshold (red) on the left plots. The right plots show that only the detector calculated by a subset filter excluding the faulty satellite (PRN12) follows a centralized $\chi^{2}$-distribution (blue) and remains under the detection threshold. The fault mode (i.e. faulty satellite) can be identified by using this fact.

Finally, Fig.18 shows Horizontal and Vertical Protection Levels (HPL \& VPL) calculated in the IM function by (19), for the four different sensor sets (with and without GPS velocity and the stereo camera), when $100 \%$ step fault percentage is introduced. These values are compared with those from a conventional snap-shot LS-RAIM applied on the GPS pseudo-range measurements. It shows that PLs are reduced and hence the navigation solution availability is improved when adding the stereo camera, and even further by the GPS velocity measurements.
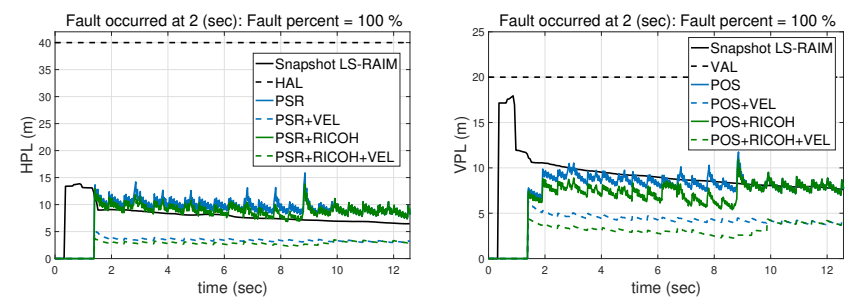

Fig. 18. Horizontal (left) and Vertical (right) Protection Levels

\section{CONCLUSION}

This paper proposed a fault tolerant navigation system for aircraft final approach by using a long-range stereo camera system. The developed stereo camera system has been tested in real time onboard the UAV, and its runway feature detection performance was validated. The tightlyintegrated vision/GNSS navigation filter with the integrity monitoring function has been evaluated on the real sensor data sets with GNSS fault emulation. Improvements both in fault detectability and in navigation performance were observed by adding the stereo camera to the conventional sensor setup. Implementation of the delayed ESKF filter on the PL software architecture is currently undergoing, towards the first closed-loop autonomous vision-aided final approach flight of K50. The challenge will be the onboard implementation and real-time execution of the IM funcion which runs a bank of subset filters in parallel.

\section{ACKNOWLEDGMENT}

This work has received funding from the European Union's Horizon 2020 research and innovation programme under grant agreement No. 690811 and the Japan New Energy and Industrial Technology Development Organization under grant agreement No. 062800, as a part of the EU/Japan joint research project entitled "Validation of Integrated Safety-enhanced Intelligent flight cONtrol (VISION)."

\section{REFERENCES}

[1] H2020 VISION: http://w3.onera.fr/h2020_vision

[2] L.Coutard and F.Chaumette and J.M.Pflimlin, Automatic Landing on Aircraft Carrier by Visual Servoing, IEEE International Conference on Intelligent Robots and Systems, 2011.

[3] V.Gibert and L.Burlion and A.Chriette and J.Boada and F.Plestan, Nonlinear Observers in Vision System: Application to Civil Aircraft Landing, IEEE European Control Conference, 2015.

[4] M. Lailacker, K. Kondak, M. Schwarzbach and T. Muskardin, Vision Aided Automatic Landing System for Fixed Wing UAV, IEEE/RSJ International Conference on Intelligent Robots and Systems, 2013.

[5] M.E.Kügler and N.C.Mumm and F.Holzapfel and A.Schwithal and M.Angermann, Vision-Augmented Automatic Landing of a General Aviation Fly-by-Wire Demonstration, AIAA SciTech Forum, 2019.

[6] T.D. Larsen, N.A. Andersen, O. Ravn and N.K. Poulsen, Incorporation of Time Delayed Measurements in a Discrete-time Kalman Filter, IEEE Conference on Decision and Control, 1998.

[7] Y.Watanabe and A.Manecy and A.Hiba and S.Nagai and S.Aoki, Vision-integrated Navigation System for Aircraft Final Approach in case of GNSS/SBAS or ILS Failures, AIAA SciTech Forum, 2019.

[8] M.Joerger and B.Pervan, Kalman Filter-Based Integrity Monitoring Against Sensor Faults, AIAA Journal of Guidance Control and Dynamics, 36(2), 2013.

[9] J. Sola, Quaternion kinematics for the error-state Kalman filter, CNRS/LAAS Technical notes, 2017.

[10] P. Viola, Rapid Object Detection using a Boosted Cascade of Simple Features, IEEE Computer Society Conference on Computer Vision and Pattern Recognition, 2001.

[11] M.A. Fischler and R.C. Bolles, Random Sample Consensus: A Paradigm for Model Fitting with Applications to Image Analysis and Automated Cartography, Comm. ACM, 381-395, 1981.

[12] M.Joerger and F.C.Chan and B.Pervan, Solution Separation Versus Residual-Based RAIM, NAVIGATION: Journal of The Institute of Navigation, 61(4), 2014

[13] C. Lesire, D. Doose and H. Cassé, Mauve: a Componentbased Modeling Framework for Real-time Analysis of Robotic Applications, IEEE ICRA Workshop on Software Development and Integration in Robotics, 2012. https://gitlab.com/ MAUVE/mauve_toolchain/wikis/home

[14] ICAO, Global Navigation Satellite System (GNSS) Manual, Doc 9849 AN/457, 2005. 